\title{
Translational control of gene expression via interacting feedback loops
}

\author{
Liang Wang \\ Division of Mathematics, School of Science and Engineering, University of Dundee, Dundee DD1 4HN, United Kingdom \\ M. Carmen Romano \\ SUPA, Institute for Complex Systems and Mathematical Biology, Department of Physics, Aberdeen AB24 3UE, United Kingdom \\ and Institute of Medical Sciences, University of Aberdeen, Foresterhill, Aberdeen AB24 3FX, United Kingdom \\ Fordyce A. Davidson ${ }^{*}$ * \\ Division of Mathematics, School of Science and Engineering, University of Dundee, Dundee DD1 4HN, United Kingdom
}

(Received 12 March 2019; published 26 November 2019)

\begin{abstract}
Translation is a key step in the synthesis of proteins. Accordingly, cells have evolved an intricate array of control mechanisms to regulate this process. By constructing a multicomponent mathematical framework we uncover how translation may be controlled via interacting feedback loops. Our results reveal that this interplay gives rise to a remarkable range of protein synthesis dynamics, including oscillations, step change, and bistability. This suggests that cells may have recourse to a much richer set of control mechanisms than was previously understood.
\end{abstract}

DOI: 10.1103/PhysRevE.100.050402

Introduction. Control of gene expression refers to the processes by which the production of proteins is regulated by the cell. This is at the heart of the functioning of all living organisms and it allows cells to adapt to their environment. Control of gene expression can occur at multiple levels $[1,2]$. In this Rapid Communication, we focus on translational control.

Translation is the process by which a protein is made from a messenger RNA (mRNA) molecule. An mRNA consists of a sequence of codons, each coding for a certain amino acid. Translation is performed by molecular machines called ribosomes, which bind to the beginning of the mRNA [5' untranslated region ( $5^{\prime}$ UTR)], scan it for the start codon, and hop from one codon to the next, thereby producing the chain of amino acids which form the protein. When the ribosome reaches the stop codon, the protein is complete, is released into the cytoplasm, and the ribosome binds off the mRNA.

Recent years have witnessed an explosion of information about how translational mechanisms regulate protein levels [3]. Prominent examples include translational control during cell stress [4], switching in the mechanism responsible for translation initiation during the cell cycle [5], and translational repression by microRNAs (miRNAs) [6-9]. One of the main advantages of translational control is that allows for a rapid cell response [10].

In this Rapid Communication, we focus on one important case of translational control that has remained unexplored within this research framework, namely, the interplay between positive and negative regulatory mechanisms. Proteins that bind to the $5^{\prime}$ UTR region of mRNAs and hinder translation play an important role in the regulation of gene expression

\footnotetext{
*f.a.davidson@dundee.ac.uk
}

[11-13]. Examples include proteins involved in neural differentiation and plasticity [14], cognitive problems [15], cell cycle and differentiation of mammalian cells [16,17], and DNA repair [18]. On the other hand, virtually all mRNAs are subject to positive feedback via ribosome recycling due to their pseudocircular structure [19,20]. A particularly pertinent example is the poly(A) binding protein (PABP) [21-25]. PABP binds to the $3^{\prime}$ end [poly(A) tail] of all mRNAs and strongly affects both translational efficiency and stability of all transcripts. Moreover, PABPs interact with the initiation factors bound to the cap of the mRNA, facilitating the circularization of the mRNA and consequent recycling of ribosomes $[19,26]$. Finally, PABPs protect the mRNA from degradation [27]. Therefore, PABPs exert control on protein synthesis via a twofold mechanism, and as a consequence, the regulation of PABP availability provides a global mode of translational regulation.

Here, we show that the interplay between negative translational feedback and ribosome recycling gives rise to a range of dynamical behavior in protein synthesis, including oscillations, step change, and bistability. The existence of at least one negative and one positive feedback loop has been proved to be a necessary condition for oscillatory behavior and multistability, respectively [28-32], but examples of this dynamical behavior have been studied mainly in transcriptional regulation [33] or coupling between transcription and translation [34].

Modeling framework. Our mathematical framework is a multicomponent model that accounts for translation, protein complex formation, and binding of protein complexes and ribosomes at the $5^{\prime}$ UTR (see Fig. 1). At its core is a stochastic model of one-dimensional transport extensively studied in nonequilibrium statistical physics - the totally asymmetric 


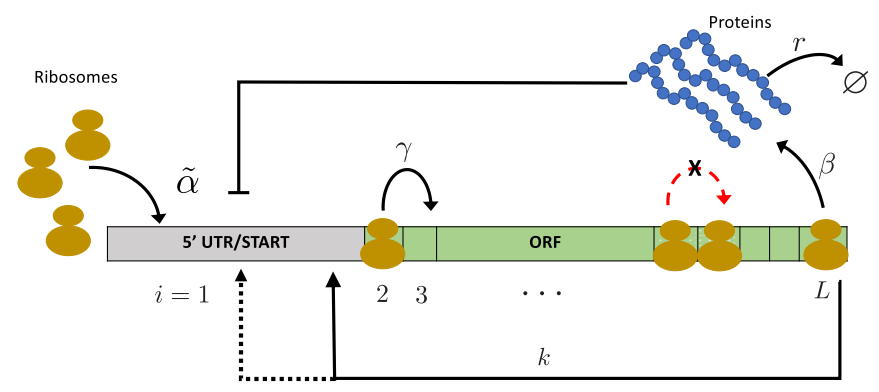

FIG. 1. Schematic of translation model with ribosome recycling and autonegative feedback. Competitive recycling (dashed line), noncompetitive recycling (solid line). Each site can be occupied by no more than one particle, so that at any time $t$ the state at site $i$ given by $S_{i}(t)$ is either $S_{i}(t)=0$ or $S_{i}(t)=1$, with $i=1, \ldots, L$, where $L$ is the lattice length. Particles bind to the first site of the lattice at rate $\tilde{\alpha}$, then hop from one site to the next at rate $\gamma$ (usually rescaled to one and done so here), and finally leave the lattice from the last site at rate $\beta$, marking the point where the associated protein synthesis is completed. See text for further details.

simple exclusion process (TASEP) [35]. Here, we augment this standard model with processes that explicitly account for protein synthesis and associated positive and negative feedback. This allows us to consider both steady-state and dynamic behaviors. Our model predicts the average number of particles per site $\rho$ and the consequent production of proteins. Ribosomes are assumed to attach at a rate $\tilde{\alpha}$ and move along the mRNA at a rate $\gamma$. On reaching the final site, ribosomes leave with rate $\beta$ and are recycled at rate $k$. At this stage a protein is produced. The protein number $N$ is controlled by a balance of synthesis and degradation with rate constant $r$. Critical to the model is that both proteins and ribosomes feed back to affect the loading rate $\tilde{\alpha}$ (see Fig. 1). We consider lattices representing realistic mRNA lengths of 500 codons and simulation times that are of the order of the half-life of typical prokaryotic mRNAs [see Supplemental Material (SM) [36]].

Translation. Ribosomes are represented by particles that hop stochastically along the sites of a one-dimensional lattice corresponding to the codons of the mRNA [37]. Note that in the standard case (setting recycling and feedback to zero here) steady-state traffic on the lattice can be classified into three main phases: low density (LD) $(\alpha<\beta, \alpha<1 / 2)$, high density (HD) $(\beta<\alpha, \beta<1 / 2)$, and the maximal current (MC) $(\alpha, \beta \geqslant 1 / 2)$. Each of these phases has an associated average density $\rho$ and current $J$ (average flux of particles), which in the limit of an infinitely long lattice are given by $\rho_{\mathrm{LD}}=\alpha, \rho_{\mathrm{HD}}=1-\beta, \rho_{\mathrm{MC}}=1 / 2$, and $J_{p}=\rho_{p}\left(1-\rho_{p}\right)$, $p \in\{\mathrm{LD}, \mathrm{HD}, \mathrm{MC}\}[38,39]$. Corresponding expressions for the modified TASEP considered here will be presented below.

Translational negative feedback. A protein can bind (often in multimeric form) to its own mRNA, thereby blocking the loading of ribosomes. Since protein binding and unbinding to the mRNA is generally much faster than ribosome loading [40], the probability of the start codon being free for ribosome loading can be described by a Hill function $f(N)=1 /\left[1+(N / \theta)^{n}\right]$, where $N$ is the protein copy number, $\theta$ measures the protein level that induces the half maximal ribosome binding rate, and $n$ measures the cooperativity of the protein multimer (see SM [36]). Thus, the intrinsic initiation rate is modified from $\alpha$ (the standard constant rate) to $\alpha f(N)$.

Translational positive feedback. The two ends of the mRNA can interact, leading to a pseudocircular structure [41], which, together with the recycling complex Rli1p [42], promote terminating ribosomes to start a new round of translation on the same mRNA [43]. Following Ref. [44], a ribosome on site $i=L$ is assumed to either detach at rate $\beta$ and enter the reservoir of free ribosomes or move directly onto site $i=1$ at a recycling rate $k$ [if $S_{1}(t)=0$ ] to reinitiate the translation process.

Protein degradation. Once synthesized, proteins enter the intracellular pool, where they are subjected to degradation. This can be a complex process $[45,46]$. However, as a detailed description is not critical to the work presented, we adopt the widely used approach of modeling removal as a Poisson process with resultant removal rate $r N$.

Finally, note that if the processes detailed above are in a steady state, then $J=r N$ and hence $N \equiv N^{*}=J / r$ and hence we can write $f=1 /\left[1+(4 I J)^{n}\right]$, where we have introduced the reciprocal factor $I:=1 /(4 \theta r)$ that measures feedback intensity (the factor of 4 is for algebraic convenience).

Models for interacting feedback loops. Experimental results suggest that recycled ribosomes are channeled downstream of the normal de novo initiation site and thus may evade the blocking effect of the protein complex [47]. This is the case discussed here and referred to as noncompetitive recycling. However, the relative position of the protein complex binding site and the recycled ribosome initiation site is not clear. Hence, an alternative is that both recycled ribosomes and $d e$ novo initiation are blocked by the protein complex (competitive recycling) (see Fig. 1). We comment on this alternative case below.

In the noncompetitive recycling case a system of ordinary differential equations that determine the dynamics of the average occupancies $\rho_{i}$ of the lattice sites is

$$
\begin{aligned}
\frac{d \rho_{1}}{d t} & =\underbrace{\alpha f(N)\left(1-\rho_{1}\right)}_{\text {de novo }}+\underbrace{k \rho_{L}\left(1-\rho_{1}\right)}_{\text {recycled }}-\rho_{1}\left(1-\rho_{2}\right) \\
\frac{d \rho_{i}}{d t} & =\rho_{i-1}\left(1-\rho_{i}\right)-\rho_{i}\left(1-\rho_{i+1}\right), \quad i=2, \ldots, L-1 \\
\frac{d \rho_{L}}{d t} & =\rho_{L-1}\left(1-\rho_{L}\right)-\beta \rho_{L}-\underbrace{k\left(1-\rho_{1}\right) \rho_{L}}_{\text {recycled }}
\end{aligned}
$$

By direct comparison with the corresponding system for the standard TASEP, effective entry and exit rates can be defined as follows,

$$
\alpha_{\mathrm{eff}}:=\alpha f(N)+k \rho_{L}, \quad \beta_{\mathrm{eff}}:=\beta+k\left(1-\rho_{1}\right) .
$$

Effective rates for the competitive recycling case can be defined equivalently (see SM [36]). However, the behavior of these two cases is similar in almost all parameter regimes and we present only the results for the noncompetitive case highlighting where any differences in the two cases arise.

Steady-state analysis of protein production and ribosome density. Setting the right-hand side of system 1 to zero and applying a mean-field approach leads to conditions that partition 
the steady states of the system into phases aligned with those for the standard case (LD, HD, and MC). These conditions are defined by direct substitution of $\alpha$ and $\beta$ with $\alpha_{\text {eff }}$ and $\beta_{\text {eff }}$, respectively, in the standard conditions stated above. In turn and after some lengthy analysis, expressions for these phases and their boundaries in terms of the system parameter are as follows - note these definitions collapse to the standard cases on setting $k=I=0$ (see SM [36]).

$$
\text { Maximal current phase: }
$$

$$
\frac{\alpha}{2 \alpha+k\left(1+I^{n}\right)} \leqslant \begin{cases}\beta \leqslant \frac{\alpha k}{1+I^{n}-2 \alpha}, & \alpha<\frac{1}{2}\left(1+I^{n}\right) \\ \beta, & \text { otherwise. }\end{cases}
$$

Within this region,

$$
\alpha_{\mathrm{eff}}=\frac{\alpha}{1+I^{n}}+\frac{k}{2\left[\beta+\sqrt{\beta\left(\beta+\frac{k}{\alpha}\left(1+I^{n}\right)\right)}\right]}
$$

and

$$
\beta_{\mathrm{eff}}=\frac{1}{2}\left[\beta+\sqrt{\beta\left(\beta+\frac{k}{\alpha}\left(1+I^{n}\right)\right)}\right] .
$$

Low-density phase:

$$
\alpha< \begin{cases}\beta\left[1+\left(\frac{4 I(\beta+k)(1-\beta)}{(1+k)^{2}}\right)^{n}\right], & 2 \beta+k<1, \\ \frac{\beta}{2 \beta+k}\left(1+I^{n}\right), & \text { otherwise. }\end{cases}
$$

Within this region, there exists a unique, positive expression for $\alpha_{\text {eff }}$ that yields unique, positive expressions for $\beta_{\text {eff }}$ and $N^{*}$.

High-density phase:

$$
\alpha> \begin{cases}\beta\left[1+\left(\frac{4 I(\beta+k)(1-\beta)}{(1+k)^{2}}\right)^{n}\right], & 2 \beta+k<1, \\ \frac{k \beta}{1-2 \beta}\left(1+I^{n}\right), & \text { otherwise. }\end{cases}
$$

Within this region, there exist either one, two, or three positive solutions, $\beta_{\text {eff }}$, that yield positive expressions for $\alpha_{\text {eff }}$ and $N^{*}$.

For $k, I>0$ and for a large range of parameter space, longrun Monte Carlo simulations of the modified TASEP reveal steady-state phases that are well characterized by Eqs. (3)-(5) (see Figs. S1 and S2 [36]). However, a deeper analysis reveals that the complex interplay of positive and negative feedback can generate distinct and different dynamical behavior.

Negative feedback and ribosome recycling induce oscillations in cellular protein level. Monte Carlo simulations reveal periodic oscillations in the number of proteins $N(t)$ within the initiation limited regime (LD phase). The stochastic nature of the individual simulations makes it difficult to systematically differentiate periodic oscillations from random fluctuations by visual inspection. However, a power spectrum analysis provides a clear demarcation: A tight, single-peaked spectrum is associated with periodic oscillations [Figs. 2(a) and 2(c)] whereas a broadband response is obtained in the case of stochastic fluctuations [Figs. 2(b) and 2(d)].
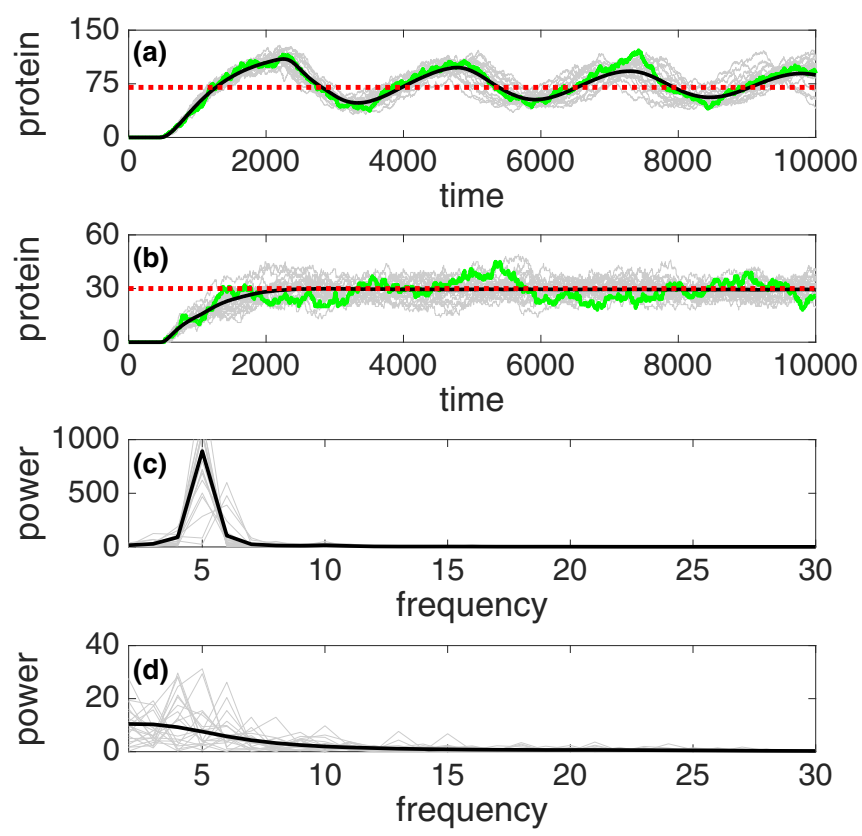

FIG. 2. Simulation and power spectrum of protein level in the low-density phase. (a), (b) Protein number as a function of time. Averages over 5000 realizations of stochastic simulations (black lines), 20 example realizations (gray lines), and single example trajectory (green line). Red dashed lines are mean field $N^{*}$ as computed using the steady-state theory. (a) Typical simulation and (c) the corresponding power spectrum from the region where oscillations are predicted to exist ( $\alpha=0.8$; note $\alpha_{\text {eff }}=0.17<0.5$, therefore corresponding to the system being in the LD phase). (b) Typical simulation and (d) the corresponding power spectrum from the region where oscillations are not predicted to occur $(\alpha=0.05)$. In all cases $\beta=0.5, k=0.2$, $I=5 / 2, r=0.002, n=5, L=500.1$ time unit $=1 / 22 \mathrm{~s}$.

The time needed for a ribosome to transit the mRNA induces a delay between initiation and completion of protein synthesis. This generates a delay in the action of the negative feedback - a mechanism known to generate oscillatory behavior [48]. A simplified model for the protein copy number $N(t)$ in the $\mathrm{LD}$ regime is

$$
\begin{aligned}
\frac{d N(t)}{d t} & =J(t)-r N(t) \\
& =\alpha_{\mathrm{eff}}(t-T)\left[1-\alpha_{\mathrm{eff}}(t-T)\right]-r N(t),
\end{aligned}
$$

where $T$ denotes the translational delay time [49]. Appealing to Eqs. (2) and setting $\rho_{L}=J(t) / \beta_{\text {eff }}$, it follows that

$$
\alpha_{\mathrm{eff}}(t)=\frac{\alpha(\beta+k)}{\alpha k+\beta\left[1+(4 \operatorname{Ir} N(t))^{n}\right]} .
$$

Substituting Eq. (7) into Eq. (6) results in a delay differential equation for $N$. The translational delay can be estimated as $T=L /\left(1-\rho^{*}\right)$, where $\rho^{*}:=\alpha_{\text {eff }}\left(N^{*}\right)$. This simplified model reproduces the amplitude and period of the stochastic simulations [cf. Figs. 2(a) and S3(d)]. It can be shown that on increasing $\alpha$ the steady state of (6) can be driven unstable via a Hopf bifurcation with the Hopf locus given by

$$
B \cos \left(\sqrt{B^{2}-r^{2}} T\right)+r=0,
$$



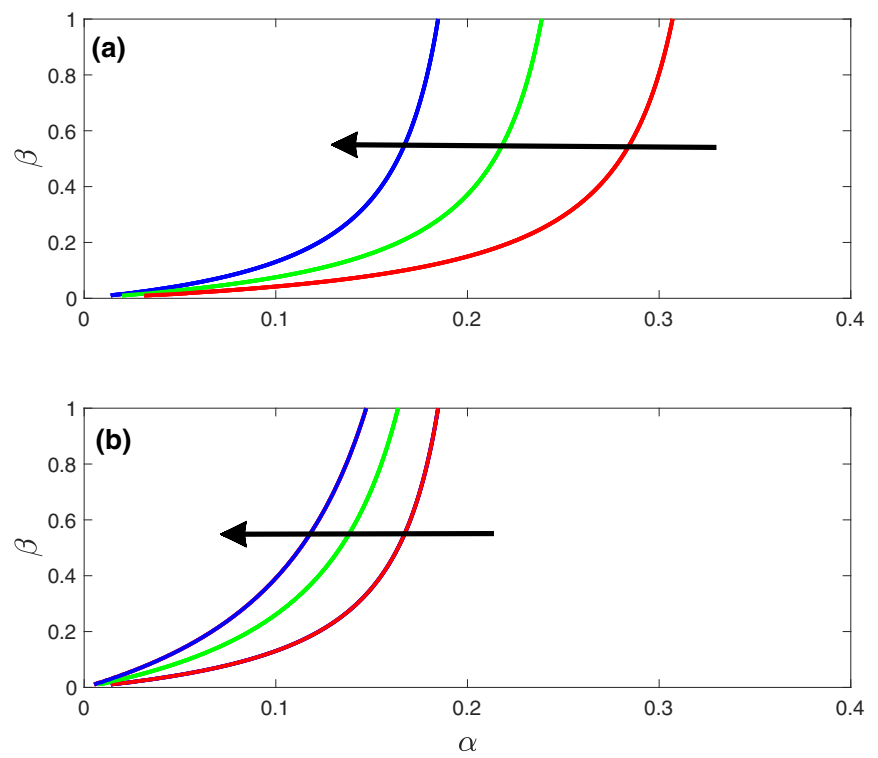

FIG. 3. The Hopf locus and the effect of varying recycling and feedback. Hopf bifurcation locus in the $\alpha-\beta$ plane computed using Eq. (8). The effect of (a) varying feedback intensity and (b) recycling is shown. Black arrows indicate the direction of increasing effect. (a) $I=25 / 14$ (red), $I=25 / 12$ (green), $I=25 / 10$ (blue) with $k=$ 0.2 and (b) $k=0.2$ (red), $k=0.4$ (green), $k=0.6$ (blue) with $I=$ $25 / 10$. $L=500$ sites, $r=0.002, n=5$.

where $B$ is a function of the system parameters (see SM [36]). This locus forms a curve in the $\alpha-\beta$ plane (see Fig. 3). After some algebra, it can be shown that necessary conditions for the existence of the Hopf locus are $n>1$ and $I>$ $F(\alpha, \beta, k, n)$ for some positive function $F$ (see SM [36]). The condition $n>1$ indicates that cooperativity in protein binding is necessary for the onset of oscillations [50]. The second condition indicates that the onset of oscillations occurs when the feedback intensity is sufficiently strong.

As one would intuitively expect, increasing feedback intensity induces the onset of oscillations at lower values of the intrinsic loading rate $\alpha$ [see Fig. 3(a)]. Interestingly, the Hopf locus also shifts left on increasing the recycling rate [see Fig. 3(b)]. Hence, counterintuitively, ribosome recycling-a positive feedback mechanism-also enhances the onset of oscillations [51].

Interplay between recycling and negative feedback induces bistability in protein production. In the MC and LD phases, the current $J$ is uniquely defined for any given parameter set. On the contrary, in the HD phase $J$ can be multiply defined: After some algebra it can be shown that $\beta_{\text {eff }}$ is the solution to the following $2 n+1$ degree equation (see SM [36]),

$$
4^{n} k I^{n} \beta \beta_{\mathrm{eff}}^{n}\left(1-\beta_{\mathrm{eff}}\right)^{n+1}-(k \beta+\alpha) \beta_{\mathrm{eff}}+\beta(\alpha+k)=0 .
$$

For $k=0$, Eq. (9) has the unique solution $\beta_{\text {eff }}=\beta$. For $I=0, \beta_{\text {eff }}$ is uniquely defined by $\beta_{\text {eff }}=\beta(\alpha+k) /(k \beta+\alpha)$ [44]. However, when both $k, I>0$, Eq. (9) can have three admissible solutions, depending on the value of $\alpha$. Thus, for suitably chosen parameters, there exists an interval of values of $\alpha$ for which three steady-state values of $N^{*}=J / r$ coexist.
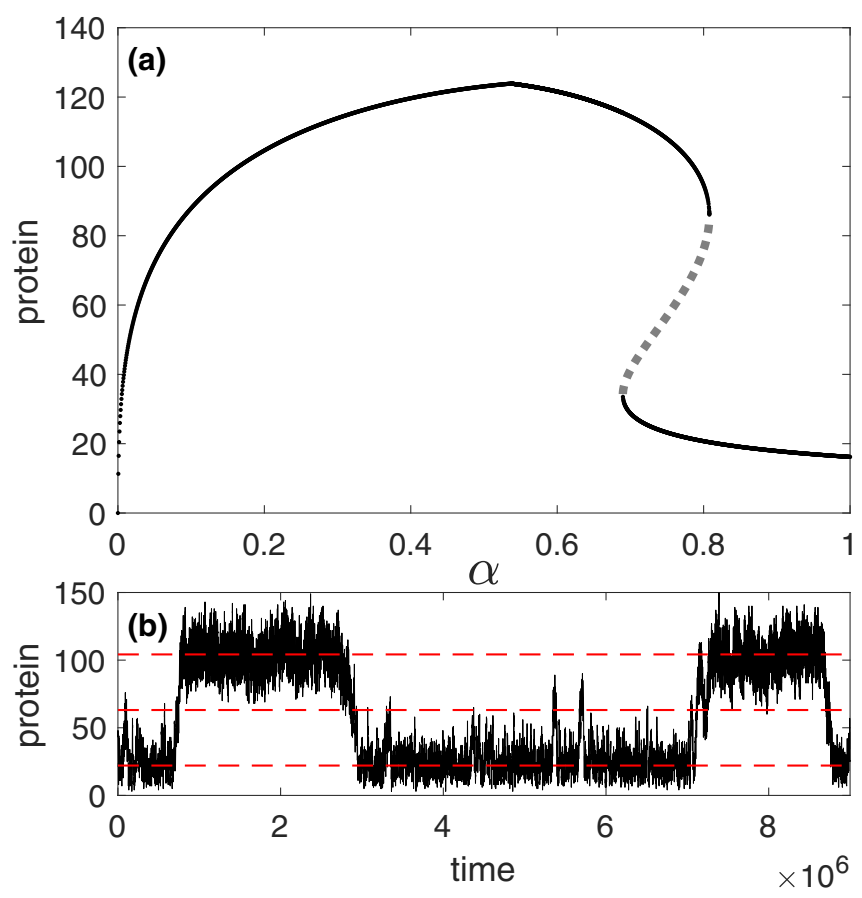

FIG. 4. (a) Protein number $N^{*}$ as a function of the initiation rate $\alpha$ as predicted by the steady-state theory. $N^{*}$ is first monotonically increasing (LD phase), then decreasing (HD phase) [44]. $N^{*}$ then passes through twofold bifurcations, leading first into, and then out of, the interval of coexistent states-with the upper and lower branches (solid black lines) separated by an intermediate state indicated (dashed gray line). (b) Monte Carlo simulation of the protein number as a function of time (solid black line) with the mean-field solutions $N^{*}$ (red dashed lines) from (a) $(\alpha=0.77)$. In both cases, $\beta=0.015, k=0.8, I=6, r=0.002, n=2, L=500$. 1 time unit $=1 / 22 \mathrm{~s}$.

Figure 4(a) shows $N^{*}$ as a function of $\alpha$, so that on increasing $\alpha$ from zero to one, the model transits from the LD to the HD phase (at $\alpha \approx 0.5$ ).

With values of $\alpha$ selected from the coexistence interval shown in Fig. 4(a), simulations reveal that the number of proteins remains centered on high or low states for a timescale orders of magnitude larger than stochastic fluctuations [Fig. 4(b)]. Rapid switching events between the favored state are accompanied by a brief hiatus at an intermediate state. The mean locations of these favored and intermediate states are well approximated by the analytic expressions for the steady states obtained from Eq. (9) [Fig. 4(a)]. Frequency histograms reveal the effect of varying $\alpha$ across the bistable region and, together with dwell-time histograms, indicate this to be a memoryless stochastic switching process (see Figs. S5 and S6).

Fixing $k$ (respectively $I$ ) and increasing $I$ (respectively $k$ ) increases the interval of values of $\alpha$ for which the fold exists (fold width-see Fig. S7). Interestingly, the location of the fold is also an increasing function of $I$ and $k$. Indeed, somewhat counterintuitively, for a fixed value of $\alpha$, increasing the intensity of the negative feedback $I$ can force the system from a low to high $N^{*}$ state. To understand this recall that within the HD regime, any change of parameters leading to a decrease in the ribosome density leads to an increase in 

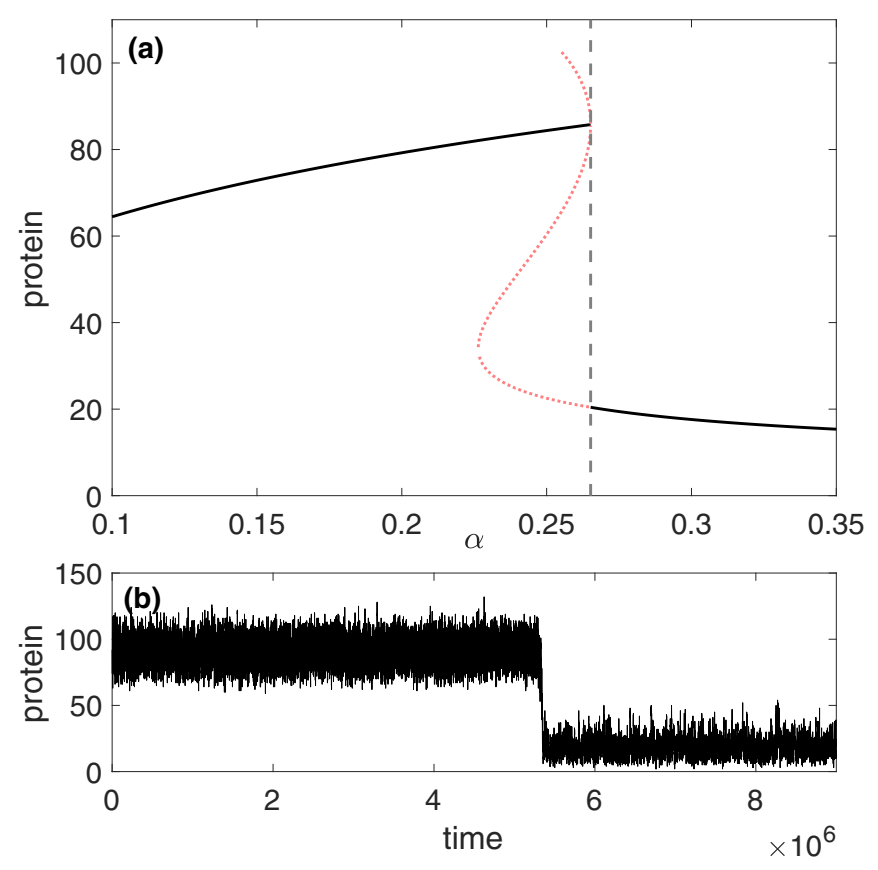

FIG. 5. Step change in steady-state protein levels. (a) Signalresponse curve for protein number $N^{*}$ as a function of the initiation rate $\alpha$ (black curves). Inadmissible solutions for (9) (red dotted curve). The LD-HD boundary (vertical gray dashed line). (B) Time series of the number of proteins: $\alpha=0.28$ then switched to $\alpha=0.3$ at time $t=4.5 \times 10^{6}$. In each case, $\beta=0.015, k=0.263, I=6$, $r=0.002, n=2, L=500$. 1 time unit $=1 / 22 \mathrm{~s}$.

the ribosomal current: Increasing $I$ decreases $\alpha_{\text {eff }}$, thereby decreasing the density of ribosomes on the mRNA. Finally, we note that bimodality in the protein production rate is a result of a careful balance between the negative and positive feedback loops and tuning one or the other can drive the system both into and out of a bimodal response (see Fig. S7). Indeed, in the case of competitive recycling no bistability occurs. Following similar calculations, it can be shown that the equation corresponding to Eq. (9) has a unique solution. It appears that feedback dominates and locks the system into a unique steady state in that case.

Feedback interplay can induce step changes in protein production. If $k$ is fixed to ensure bistability, a critical value of $I$ exists at which the right boundary of the bistable region coincides precisely with the LD-HD boundary. In this case, as the initiation rate $\alpha$ passes through the LD-HD boundary, a discontinuity in the number of proteins occurs [Fig. 5(a)]. (Qualitatively similar behavior is obtained on keeping I fixed and varying $k$.) This step change in the number of proteins can be large, suggesting that small changes in the ribosome initiation rate $\alpha$ can result in a significant shift in protein levels. Simulations confirm this theoretical prediction. On increasing $\alpha$ dynamically during a simulation, a step change (around $75 \%$ reduction) was clearly induced on crossing the $\mathrm{LD} / \mathrm{HD}$ critical value [Fig. 5(b)]. This cliff-edge response is another unique feature resulting from the interplay between feedback and recycling.

Conclusions. Our model shows the rich dynamical behavior caused by the interplay of negative and positive feedback loops in translation, and it provides a general mathematical framework to analyze other kinds of gene expression regulation, such as regulation exerted by miRNAs [52]. It suggests that this interplay could provide cells with a versatile mechanism to adapt their protein levels according to the environment. The centrally important protein PABP is subject to ribosome recycling and is known to exhibit translational negative feedback. Interestingly, PABP has also been implicated in circadian oscillations [53]. Finally, disturbances of poly(A) tail length have been linked to a number of physiological and pathological processes. Therefore, a better understanding of the interplay of ribosome recycling and translational negative feedback has far-reaching consequences.

Acknowledgments. L.W. was supported by the Northern Research Partnership.
[1] J. Hausser, A. Mayo, L. Keren, and U. Alon, Nat. Commun. 10, 68 (2019).

[2] U. Alon, An Introduction to Systems Biology: Design Principles of Biological Circuits (Chapman \& Hall/CRC, Boca Raton, FL, 2007).

[3] J. Hershey, N. Sonenberg, and M. Mathews, Cold Spring Harbor Perspect. Biol. 4, a011528 (2012).

[4] K. Spriggs, M. Bushell, and A. Willis, Mol. Cell 40, 228 (2014).

[5] S. Pyronnet, J. Dostie, and N. Sonenberg, Genes Dev. 15, 2083 (2001).

[6] M. Selbach, B. Schwanhäusser, N. Thierfelder, Z. Fang, R. Khanin, and N. Rajewsky, Nature (London) 455, 58 (2008).

[7] A. Zinovyev, N. Morozova, A. N. Gorban, and A. Harel-Belan, Mathematical modeling of microrna-mediated mechanisms of translation repression, in MicroRNA Cancer Regulation: Advanced Concepts, Bioinformatics and Systems Biology Tools, edited by U. Schmitz, O. Wolkenhauer, and J. Vera (Springer, Dordrecht, 2013), pp. 189-224.
[8] S. Nikolov, J. V. Gonzalez, M. Nenov, and O. Wolkenhauer, Biotechnol. Biotechnol. Equip. 26, 3315 (2012).

[9] A. Zinovyev, N. Morozova, N. Nonne, E. Barillot, A. HarelBellan, and A. N. Gorban, BMC Syst. Biol. 4, 13 (2010).

[10] J. W. Hershey, N. Sonenberg, and M. B. Mathews, Cold Spring Harbor Perspect. Biol., doi:10.1101/cshperspect.a032607 (2018).

[11] K. Abdelmohsen, Modulation of Gene Expression by RNA Binding Proteins: mRNA Stability and Translation Fundamental Principles of Optical Lithography (InTech Open, London, 2012).

[12] O. Levi and Y. Arava, PLoS Biol. 17, 1 (2019).

[13] R. Betney, J. de Silva, and I. Stansfield, RNA-A 16, 655 (2010).

[14] M. N. Hinman and H. Lou, Cell Mol. Life Sci. 65, 3168 (2008).

[15] G. J. Bassell and S. T. Warren, Neuron 60, 201 (2008).

[16] C. K. Damgaard and J. Lykke-Andersen, Genes Dev. 25, 2057 (2011). 
[17] V. Dormoy-Raclet, J. Markovits, A. Jacquemin-Sablon, and H. Jacquemin-Sablon, RNA Biol. 2, 112 (2005).

[18] T. Fukuda, M. Ashizuka, T. Nakamura, K. Shibahara, K. Maeda, H. Izumi, K. Kohno, M. Kuwano, and T. Uchiumi, Nucleic Acids Res. 32, 611 (2004).

[19] S. Wells, P. Hillner, R. Vale, and A. Sachs, Mol. Cell 2, 135 (1998).

[20] Z. A. Afonina, A. G. Myasnikov, V. A. Shirokov, B. P. Klaholz, and A. S. Spirin, Nucleic Acids Res. 42, 9461 (2014).

[21] E. Hornstein, H. Harel, G. Levy, and O. Meyuhas, FEBS Lett. 457, 209 (1999).

[22] O. Neto, N. Standart, and C. Desa, Nucleic Acids Res. 23, 2198 (1995).

[23] J. Bag, J. Biol. Chem. 276, 47352 (2001).

[24] G. Patel, S. Ma, and J. Bag, Nucleic Acids Res. 33, 7074 (2005).

[25] B. Gorgoni and N. Gray, Briefings Funct. Genomics Proteomics 3, 125 (2004).

[26] A. Kahvejian, Y. V. Svitkin, R. Sukarieh, M. N. M'Boutchou, and N. Sonenberg, Genes Dev. 19, 104 (2005).

[27] D. Cao and R. Parker, RNA 7, 1192 (2001).

[28] J. L. Gouz, J. Biol. Syst. 06, 11 (1998).

[29] E.-H. Snoussi, J. Biol. Syst. 06, 3 (1998).

[30] P. François and V. Hakim, Proc. Natl. Acad. Sci. USA 101, 580 (2004).

[31] L. Potvin-Trottier, N. Lord, G. Vinnicombe, and J. Paulsson, Nature (London) 538, 514 (2016).

[32] E. Ullner, A. Koseska, J. Kurths, E. Volkov, H. Kantz, and J. García-Ojalvo, Phys. Rev. E 78, 031904 (2008).

[33] R. Hermsen, B. Ursem, and P. R. ten Wolde, PLoS Comput. Biol. 6, 1 (2010).

[34] Y. Zarai and T. Tuller, PLoS Comput. Biol. 14, 1 (2018).

[35] B. Derrida, E. Domany, and D. Mukamel, J. Stat. Phys. 69, 667 (1992).

[36] See Supplemental Material at http://link.aps.org/supplemental/ 10.1103/PhysRevE.100.050402 for supporting analysis and numerical simulations.
[37] C. MacDonald, J. Gibbs, and A. Pipkin, Biopolymers 6, 1 (1968).

[38] B. Derrida, M. Evans, C. Hakim, and V. Pasquier, J. Phys. A: Math. Gen. 26, 1493 (1993).

[39] G. Schütz and E. Domany, J. Stat. Phys. 72, 277 (1993).

[40] L. Chen, R. Wang, T. Kobayashi, and K. Aihara, Phys. Rev. E 70, 011909 (2004).

[41] D. Barthelme, S. Dinkelaker, S.-V. Albers, P. Londei, U. Ermler, and R. Tampe, Proc. Natl. Acad. Sci. USA 108, 3228 (2011).

[42] C. Shoemaker and R. Green, Proc. Natl. Acad. Sci. USA 108, 1392 (2011).

[43] N. Amrani, S. Ghosh, D. Mangus, and A. Jacobson, Nature (London) 435, 1276 (2008).

[44] E. Marshall, I. Stansfield, and M. Romano, J. R. Soc. Interface 11, 104 (2014).

[45] D. A. Rothenberg, M. J. Taliaferro, S. M. Huber, T. J. Begley, P. C. Dedon, and F. M. White, iScience 9, 367 (2018).

[46] R. Golan-Lavi, C. Giacomelli, G. Fuks, A. Zeisel, J. Sonntag, S. Sinha, W. Köstler, S. Wiemann, U. Korf, Y. Yarden, and E. Domany, Cell Rep. 18, 3129 (2017).

[47] L. Rajkowitsch, C. Vilela, K. Berthelot, C. Ramirez, and J. McCarthy, J. Mol. Biol. 335, 71 (2004).

[48] S. Pigolotti, S. Krishna, and M. Jensen, Proc. Natl. Acad. Sci. USA 104, 6533 (2007).

[49] Note that in agreement with numerical simulations, we only expect oscillations within the LD phase, since in both HD and $\mathrm{MC}$, the current $J$ is independent of the delay between loading and exit (see SM [36]).

[50] M. Elowitz and S. Leibler, Nature (London) 403, 335 (2000).

[51] B. Ananthasubramaniam and H. Herzel, PLoS One 2014, e104761 (2014).

[52] S. Oliveto, M. Mancino, N. Manfrini, and S. Biffo, World J. Biol. Chem. 8, 45 (2017).

[53] S. Kojima, E. Sher-Chen, and C. Green, Genes Dev. 26, 2724 (2012). 